\title{
Retroperitoneal primary mucinous adenocarcinoma: A case report
}

\author{
HAIPING JIANG ${ }^{1}$, KETAO JIN ${ }^{2,4}$, QIHAN YOU ${ }^{3}$, WEIJIA FANG ${ }^{1}$ and NONG XU ${ }^{1}$ \\ Departments of ${ }^{1}$ Internal Oncology, ${ }^{2}$ Surgical Oncology and ${ }^{3}$ Pathology, First Affiliated Hospital, \\ College of Medicine, Zhejiang University, Hangzhou; ${ }^{4}$ Department of Surgery, \\ Affiliated Zhuji Hospital, Wenzhou Medical College, Zhuji, Zhejiang, P.R. China
}

Received February 23, 2011; Accepted May 12, 2011

DOI: $10.3892 / \mathrm{ol} .2011 .319$

\begin{abstract}
Retroperitoneal primary mucinous adenocarcinoma (RPMA) is extremely rare and the histogenesis of this tumor remains unclear. There is no consensus on the appropriate treatment for RPMA. Surgical resection is standard for the treatment of RPMA, whereas the benefits of chemotherapy with regard to this tumor remain to be established. This case report concerns a 21-year-old female individual with RPMA. The patient initially presented with chronic lower back pain and weight loss. Additionally, carcinoembryonic antigen (CEA) levels were found to be elevated. A computed tomography scan revealed a mass in the abdominal cavity. Consequently, laparotomy was performed, which revealed a well-defined tumor in the right retroperitoneum. Chemotherapy as a monotherapy was not considered as a viable treatment option. Therefore, the patient was initially administered a combined treatement of oxaliplatin and 5-fluorouracil. This treatment was then changed to paclitaxel and 5-fluorouracil. Findings showed a decrease in the CEA serum levels, indicating that this combination treatment may be efficacious in the treatment of RPMA since local recurrence following surgical resection was well controlled with chemotherapy.
\end{abstract}

\section{Introduction}

Retroperitoneal primary mucinous adenocarcinoma (RPMA) is extremely rare and the histogenesis of this tumor remains unknown. As with most retroperitoneal masses, RPMA causes clinical symptoms or is perceived by patients only when the mass grows to a sufficiently large size. Laboratory studies lack the appropriate levels of specialization for this tumor and imaging methods merely reveal cystic lesions, neither

Correspondence to: Dr Nong Xu, Department of Internal Oncology, First Affiliated Hospital, College of Medicine, Zhejiang University, 79 Qingchun Road, Hangzhou, Zhejiang 310003, P.R. China E-mail: xunonghz@163.com

Key words: retroperitoneum, mucinous adenocarcinoma, chemotherapy of which result in accurate diagnosis. Surgical resection is standard for the treatment of RPMA, whereas chemotherapy for this tumor has not been rendered an efficacious treatment modality. This case study reports a 21-year-old woman with RPMA. Following laparotomy, combined treatments were administered and the benefits thereof were investigated.

\section{Case report}

A 21-year-old woman presented with chronic lower back pain and weight loss for a period of 10 months. Her physical examination did not present any irregularities. She had no significant past medical histology or family history of disease. Laboratory data showed high levels of the carcinoembryonic antigen (CEA) $(338.39 \mathrm{ng} / \mathrm{ml})$ and carbohydrate antigen (CA) 19-9 (253.13 U/ml). A computed tomography (CT) scan revealed a mass measuring approximately $14.6 \times 7.7$ $\mathrm{cm}$ in the abdominal cavity with enlarged lymph nodes along the pancreas. There was a low-density area inside the mass, which was slightly heterogeneously enhanced (Fig. 1). Following a laparotomy, an adult fist-sized well-defined tumor was observed in the right retroperitoneum, which was covered with intact peritoneum. No ascites were noted, and the liver and kidneys appeared normal and were medially displaced. During surgical resection the mass, which consisted of multiloculated cyst with abundant intracytoplasmic mucin was ruptured. The microscopic examination confirmed a mucinous adenocarcinoma (Fig. 2A) and tumor cells were positive for caudal-related homeodomain protein 2 (CDX2) (Fig. 2B), cytokeratin 20 (Fig. 2C) and cytokeratin 19 (Fig. 2D), but negative for CA125 (Fig. 2E), and the estrogen and progesterone receptors (ER/PR) (Fig. 2F). The patient returned 4 months after the operation with elevated levels of CEA (970 ng/ml) and CA19-9 (1762 U/ml). A CT scan revealed local recurrences in the retroperitoneum (Fig. 3A). After receiving intravenous oxaliplatin and 5-fluorouracil (5-FU) for 3 cycles, no change was evident in the CEA and CA19-9 levels, and the CT scans revealed a slightly larger tumor (Fig. 3B). The regimen was then switched to 5-FU and paclitaxel. Following 4 cycles of 5-FU and paclitaxel, the CEA and CA19-9 levels decreased to $313 \mathrm{ng} / \mathrm{ml}$ and $272.5 \mathrm{U} / \mathrm{ml}$, respectively. During the regular follow-up, the tumor remained stable (Fig. 3C). 


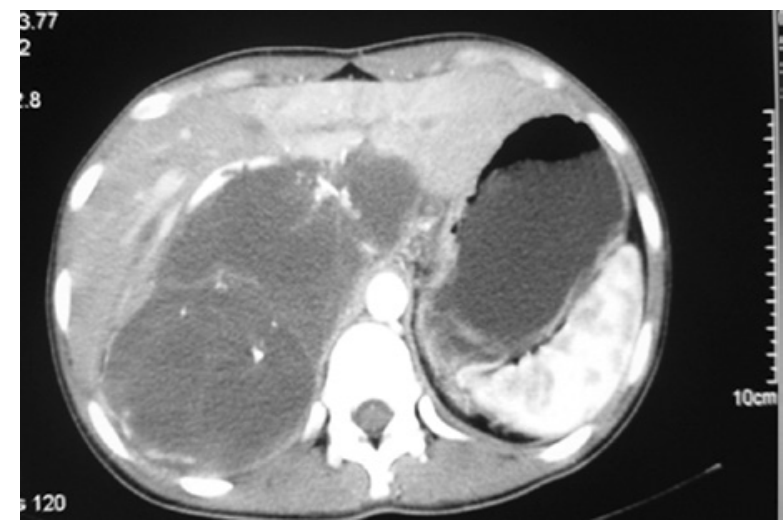

Figure 1. A computed tomography scan revealed a mass that measured approximately $14.6 \times 7.7 \mathrm{~cm}$ in the abdominal cavity with enlarged lymph nodes along the pancreas. There was a low-density area inside the mass, and the mass was slightly heterogeneously enhanced.

\section{Discussion}

RPMA is a rare phenomenon, as indicated by the few cases reported since it was first described in 1977 by Roth (1). No other reported cases of RPMA in Chinese women are available in the English literature.

Due to its rarity, the histogenesis of RPMA remains to be determined and four main hypotheses have been proposed to explain the histogenic origin of the tumor. One hypothesis suggests that the tumor arises from a teratoma with predominant mucinous epithelium (2), whereas other authors postulate that it is caused by intestinal duplication, also known as enterogenous genesis (3). The intestinal-like epithelioma surrounding the cystic tumors in our case potentially support this hypothesis. The third hypothesis supports that the tumor arises from heterotopic ovarian tissue. However, no records exist pertaining to ovarian tissue in RPMA $(4,5)$, and in our case the ovaries were normal. Previously, a fourth hypothesis

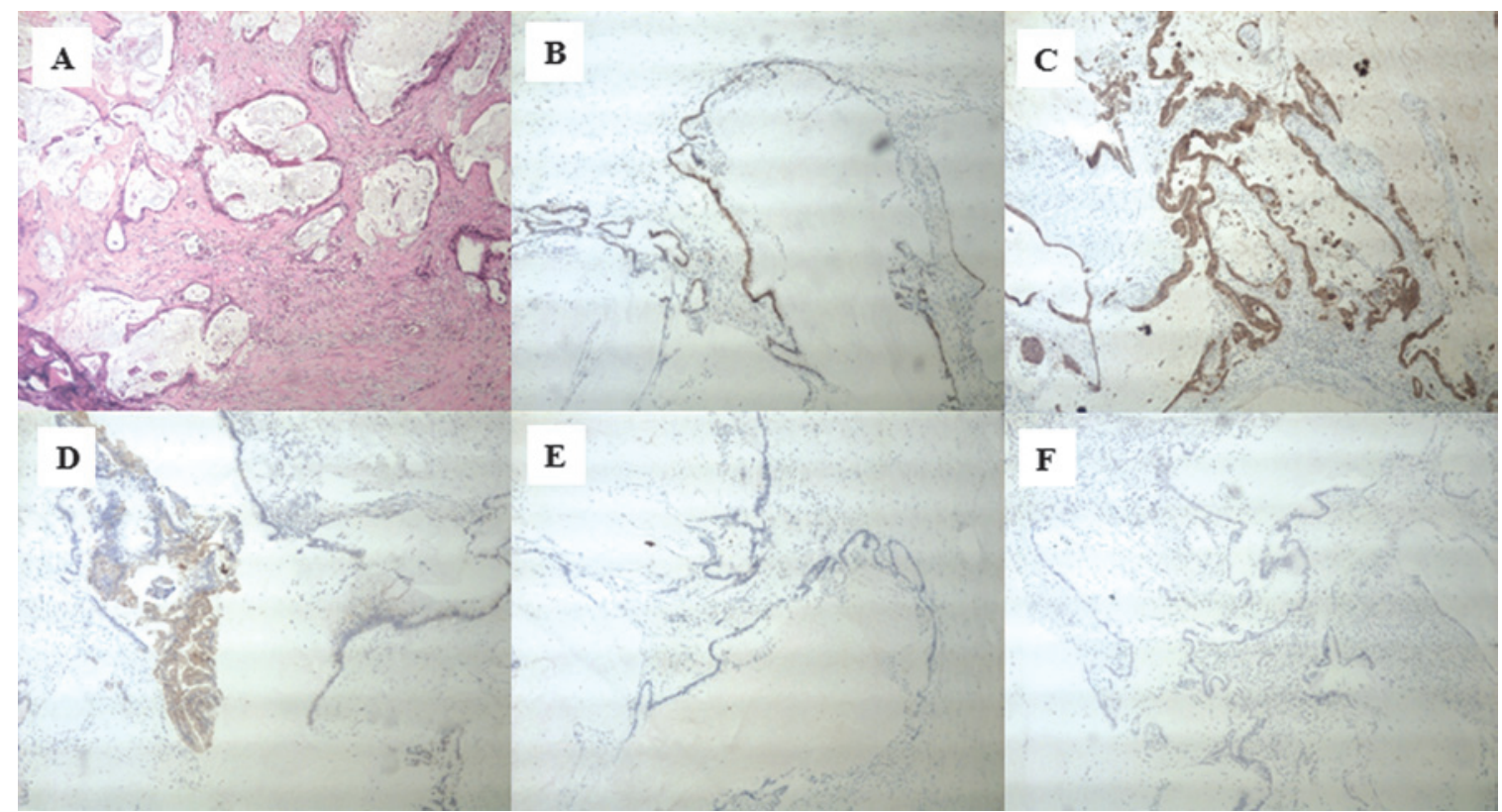

Figure 2. The microscopic examination confirmed (A) a mucinous adenocarcinoma. Tumor cells were positive for (B) the caudal-related homeodomain protein 2 (CDX2), (C) cytokeratin 20 and (D) cytokeratin 19, but negative for (E) CA125 and (F) estrogen and progesterone receptors (ER/PR). Magnification, $\mathrm{x} 400$.

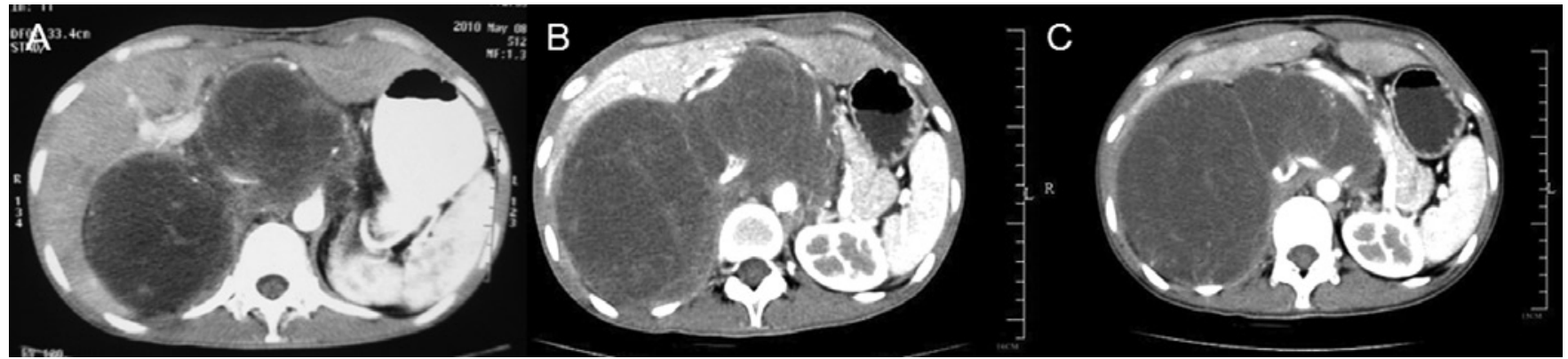

Figure 3. A computed tomography (CT) scan revealed local recurrences in the (A) retroperitoneum. The CT scan revealed a slightly larger tumor following chemotherapy with (B) intravenous oxaliplatin and 5-fluorouracil (5-FU) for 3 cycles. The tumor was stable after (C) chemotherapy with 5-FU and paclitaxel for 4 cycles. 
became widely accepted, which suggests that tumors arise from invagination of the peritoneal epithelium and undergo metaplasia during embryonic growth (6).

RPMA occurs almost exclusively in women, with the exception of 4 male cases reported in the literature (7-10). RPMA is usually observed in middle-aged individuals, although patient ages have ranged from 17 to 86 years. In the latter cases, the mass was usually large, ranging from 10 to more than $20 \mathrm{~cm}$ in diameter. According to the literature, RPMA symptoms are non-specific, with the most common ones being abdominal discomfort and palpable asymptomatic mass.

Preoperative diagnosis of RPMA is difficult, as tumor markers such as CA-125, CEA and CA19-9 may not increase and may lack specificity, thereby making the exact origin of the lesion from other tumors, such as ovarian cyst, cystic mesothelioma, cystic lymphangioma, non-pancreatic pseudocyst and renal cyst, difficult to pinpoint. However, Tangjitgamol et al hypothesized that tumor markers may help in determining a recurrent tumor, such as colon cancer (11). In our case, CEA and CA19-9 reached levels of $970 \mathrm{ng} / \mathrm{ml}$ and $1762 \mathrm{U} / \mathrm{ml}$, respectively, 4 months after sugery and a CT scan confirmed local recurrences in the retroperitoneum. After receiving 5-FU and paclitaxel, the serum CEA and CA19-9 levels in the patient decreased. Ultrasonography, CT and magnetic resonance imaging are often used to localize the tumor. However, these methods cannot easily differentiate between a benign and a malignant neoplasm (12). Yang et al suggested that when encountering a cystic lesion with the characteristic of displacing the colon, kidney or ureter medially, surgeons should include RPMA in the preoperative diagnosis (13). Needle biopsy may also be an unreliable method with which to diagnose this tumor, since it is not effective in determining malignancy in cystic tumors.

Laparotomy is necessary to facilitate accurate decisionmaking and treatment. Investigators are in agreement regarding the complete removal of the lesion. However, how extensive the surgery should be remains controversial. Given the assumption that RPMA occurs in heterotopic ovarian tissue, Lee et al recommended total hysterectomy as well as oophorectomy (14). On the other hand, Kessler et al suggested that hysterectomy and salpingo-oophorectomy are not suitable for the treatment of RPMA if the uterus and ovaries are macroscopically normal (4). Moreover, Law et al advocated laparoscopic excision of the tumor, thereby sparing fertility in these women (15). In our case, we resected the tumor, since the uterus and ovaries were relatively normal in appearance, and the patient hoped to remain fertile.

Chemotherapy for RPMA is not well established as the benefits of adjuvant chemotherapy have yet to be established. Lee et al (16) reported 5 patients who were administered with adjuvant chemotherapy following resection. Of these patients, 1 developed paraovarian recurrence despite undergoing cytoxan chemotherapy for 21 months, while 2 succumbed to widespread metastasis 4 and 18 months after surgery (16). Certain authors have suggested that since RPMA had similar mechanisms in its histogenesis to the ovarian mucinous tumor, chemotherapy should be administered, as in the case of this latter tumor. Paclitaxel with cisplatin or carboplatin combination chemotherapy may also be effective. Tenti et al reported 2 cases of RPMA with cystic rupture; the patient who underwent adjuvant chemotherapy was free of tumors for 33 months postoperatively (17). Kessler et al recommended that chemotherapy should be performed in the cases whose tumor was ruptured during surgery or had invaded to adjacent structures $(4,15)$. In our case, the tumor was ruptured during surgery and the patient experienced a recurrence 4 months after operation. The patient received chemotherapy with intravenous oxaliplatin and 5-FU for 3 cycles based on the intestinal-like epithelioma surrounding the cystic tumors. The CEA and CA19-9 serum levels stopped increasing, whereas the tumor increased slightly in size. To obtain a greater efficacy, the regimen was switched to 5-FU and paclitaxel for 4 cycles, resulting in decreased serum levels of CEA (313 ng/ml) and CA19-9 (272.5 U/ml) and a stable tumor. Thus, our clinical experience indicated that 5-FU and paclitaxel may be effective for RPMA.

In conclusion, RPMA is a rare tumor and usually presents with an asymptomatic abdominal mass. Preoperative diagnosis of RPMA remains difficult and surgeons should be aware of this tumor when encountering a large retroperitoneal cystic mass. Treatment of RPMA remains controversial. Extirpative surgery is currently the standard treatment, since the role of chemotherapy for the treatment of RPMA has yet to be determined. Further studies are therefore required to establish optimal treatment protocols for this rare neoplasm.

\section{References}

1. Roth LM and Ehrlich CE: Mucinous cystadenocarcinoma of the retroperitoneum. Obstet Gynecol 49: 486-488, 1977.

2. Papadogiannakis N, Gad A and Ehliar B: Primary retroperitoneal mucinous tumor of low malignant potential: histogenetic aspects and review of the literature. Acta Pathol Microbiol Immunol Scand Suppl 105: 483-486, 1997.

3. Thorbeck VC, Gustein D, Salvi M and Plata J: Retroperitoneal enteroid cystadenocarcinoma (possible intestinal origin). Rev Esp Enferm Apar Dig (In Spanish) 66: 329-334, 1984.

4. Kessler TM, Kessler W, Neuweiler J and Nachbur BH: Treatment of a case of primary retroperitoneal mucinous cystadenocarcinoma: is adjuvant hysterectomy and bilateral salpingooophorectomy justified? Am J Obstet Gynecol 187: 227-232, 2002.

5. De León DC, Pérez-Montiel D, Chanona-Vilchis J, DueñasGonzález A, Villavicencio-Valencia V and Zavala-Casas G: Primary retroperitoneal mucinous cystadenocarcinoma: report of two cases. World J Surg Oncol 5: 5, 2007.

6. Subramony C, Habibpour S and Hashimoto LA: Retroperitoneal mucinous cystadenoma. Arch Pathol Lab Med 125: 691-694, 2001.

7. Green JM, Bruner BC, Tang WW and Orihuela E: Retroperitoneal mucinous cystadenocarcinoma in a man: case report and review of the literature. Urol Oncol 25: 53-55, 2007.

8. Thamboo TP, Sim R, Tan SY and Yap WM: Primary retroperitoneal mucinous cystadenocarcinoma in a male patient. J Clin Pathol 59: 655-657, 2006

9. Motoyama T, Chida T, Fujiwara T and Watanabe H: Mucinous cystic tumor of the retroperitoneum: a report of two cases. Acta Cytol 38: 261-266, 1994.

10. Hrora A, Reggoug S, Jallal H, Sabbah F, Benamer A, Alaoui M, Raiss M, Ahallat M: Primary retroperitoneal mucinous cystadenocarcinoma in a male patient: a case report. Cases Journal 2: 7196, 2009.

11. Tangjitgamol S, Manusirivithaya S, Sheanakul C, Leelahakorn S, Tha-waramsara $\mathrm{T}$ and Kaewpila N: Retroperitoneal mucinous cystadenocarcinoma: A case report and review of literature. Int J Gynecol Cancer 12: 403-408, 2002.

12. Matsubara M, Shiozawa T, Tachibana R, Hondo T, Osasda K, Kawaguchi K, Kimura K and Konishi I: Primary retroperitoneal mucinous cystadenoma of borderline malignancy: a case report and review of the literature. Int J Gynecol Pathol 24: 218-223, 2005 . 
13. Yang DM, Jung DH, Kim H, Kang JH, Kim SH, Kim JH and Hwang HY: Retroperitoneal cystic masses: CT, clinical, and pathological findings and literature review. Radiographics 24: 1353-1365, 2004.

14. Lee IW, Ching KC, Pang M and Ho TH: Two cases of primary retroperitoneal mucinous cystadenocarcinoma. Gynecol Oncol 63: 145-150, 1996.

15. Law KS, Chang TM and Tung JN: Fertility-sparing treatment of a primary retroperitoneal mucinous cystadenocarcinoma. BJOG 113: 612-614, 2006
16. Lee SA, Bae SH, Ryoo HM, Jung HY, Jang SB and Kum YS: Primary retroperitoneal mucinous cystadenocarcinoma: a case report and review of the literature. Korean J Intern Med 22: 287-291, 2007

17. Tenti P, Carnevali L, Tateo S and Durola R: Primary mucinous cystadeno-carcinoma of retroperitoneum: Two cases. Gynecol Oncol 55: 308-312, 1994. . 